\title{
Analysis of Instruments and Students' Abilities in Material Number Patterns Using the Rasch Model
}

\author{
Tia Purniati ${ }^{1}$, Turmudi ${ }^{1}$, Maya Evayanti ${ }^{2}$, Didi Suhaedi ${ }^{3}$ \\ \{tpurniati@upi.edu1, turmudi@upi.edu², mayaevayanti@yahoo.com ${ }^{3}$, dsuhaedi@unisba.ac.id ${ }^{4}$ \} \\ Departemen Pendidikan Matematika, Universitas Pendidikan Indonesia, Jl. Dr. Setiabudi No. 229, \\ Bandung 40154, Indonesia ${ }^{1}$, SMP Laboratorium Percontohan UPI, Jl. Dr. Setiabudi No. 229, Bandung \\ 40154, Indonesia ${ }^{2}$, Program Studi Matematika, Universitas Islam Bandung, Jl. Tamansari No. 1, \\ Bandung 40116, Indonesia ${ }^{3}$
}

\begin{abstract}
This study aims to analyze the instruments and abilities of students on material number patterns using the Rasch model. The instrument was tested on 29 8th grade junior high school students consisting of 15 female students and 14 male students. Instrument analysis and student ability using the Rasch model based on instrument difficulty (item measure), student ability level (person measure), Wright map (person-item map), and instrument analysis. The results showed that there was one difficult question, six medium questions, and one easy question. In addition, there were five students with high abilities, sixteen students with medium abilities, and eight students with low abilities. The level of difficulty of the diversity instruments is not far adrift, but the level of student ability varies. The interaction between students and instruments as a whole is bad. The consistency of answers from students is weak, but the quality of the instrument is sufficient.
\end{abstract}

Keywords: Number patterns, Rasch model

\section{Introduction}

Algebra is a fundamental material in school mathematics. This material is important for students to master because algebra is widely used in everyday life [1]. One of the material in algebra is the number pattern [1], [2]. Number patterns are associated with arithmetic generalizations that are generally difficult for students to master [3], [4]. To improve the quality of learning in material number patterns can be done by analyzing the instruments and abilities of students using the Rasch model.

The Rasch model was introduced by a mathematician Goerg Rasch from the University of Copenhagen, Denmark. This model deals with a group of statistical techniques that are used as a mathematical approach to assessing measurements [5]. Rasch developed a measurement model that determines the relationship between the level of student ability and the level of difficulty of the problem by using the logarithmic function to produce measurements with the same interval. The unit used in the Rasch model is a logit (log odds unit) which shows the level of student ability and the level of difficulty of the questions. So based on the logit value obtained, it was concluded that the level of success of students in working on problems depends very much on the level of student ability and the level of difficulty of the questions [6]. The Rasch model was later popularized by Benjamin Wright of the University of Chicago, United States $[7]$. 
The Rasch model can produce good and accurate measurement instruments, because the Rasch model meets five objective measurement criteria, namely: (1) providing a linear scale with the same interval, (2) can predict the missing data, (3) providing estimates more precisely, (4) capable of detecting model inaccuracies, (5) producing replicable measurements [6].

\section{Methods}

This study aims to analyze the instruments and abilities of students on material number patterns using the Rasch model. The form of the instrument used was ice say eight questions. The instrument was tested on 29 8th grade junior high school students consisting of 15 female students and 14 male students. The data collected was then analyzed using Winsteps software version 4.4.5. The Rasch analysis model used is the instrument difficulty level analysis (item measure), student ability level analysis (person measure), Wright map analysis (person-item map) and instrument analysis.

\section{Result and discussion}

Here are the results of the analysis of instruments and students' abilities on material number patterns using the Rasch model based on the level of difficulty of the instrument (item measure), the level of student ability (person measure), Wright map (person-item map), and instrument analysis

\subsection{Instrument difficulty level (Item Measure)}

Analysis of the difficulty level of the instrument is useful to find out the problems that are classified as difficult, moderate, and easy. Analysis of the level of difficulty of the instrument in the material number patterns can be seen in Figure 1 through detailed logit information (measure) of each question. Data are displayed sequentially from the largest logit value to the smallest. A large logit value indicates a high level of problem difficulty (difficult), while a small logit value indicates a low level of problem difficulty (easy). If the logit values are the same, this shows the difficulty level of the problem is the same [6]. 


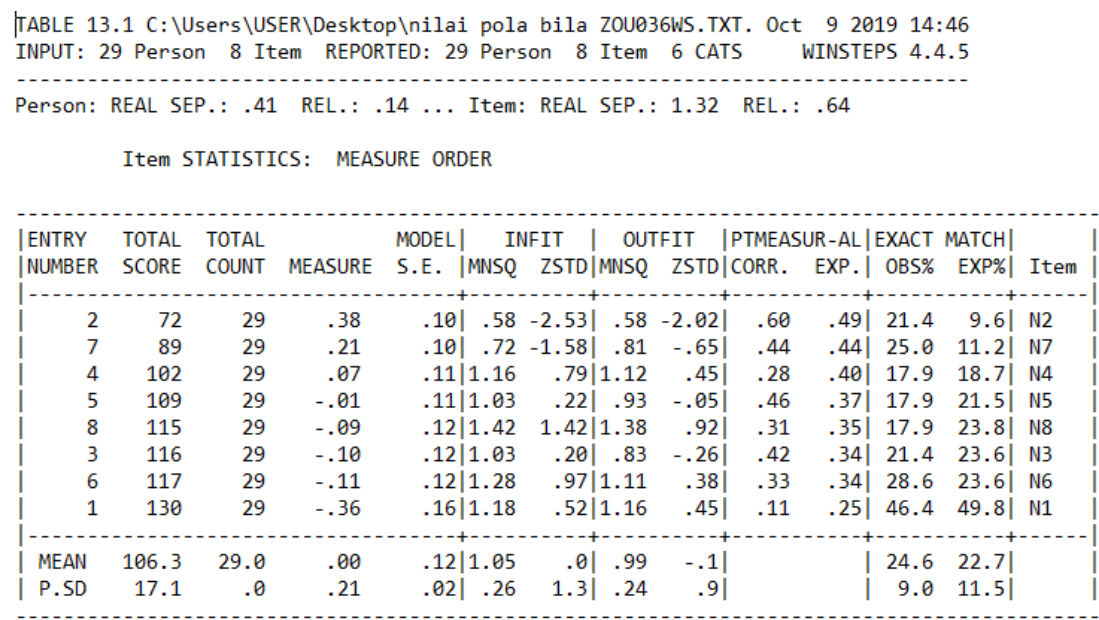

Fig. 1. Instrument Difficulty Level.

Based on Figure 1, the question that has the highest level of difficulty (difficult) is the N2 question with a logit value of +0.38 with a total score of 72 . This is because the $\mathrm{N} 2$ question requires students to make generalizations from the sequence of numbers. Generalizing rows of numbers is one part of arithmetic generalizations, which are generally difficult for students to do [9]. While the questions that have the lowest level of difficulty (easy) are questions N1 with a logit value of -0.36 with a total score of 130. Questions N3 and N6 have almost the same logit value, namely -0.10 and -0.11 , respectively this shows the difficulty level of the two questions is almost the same.

The resulting scale has the same distance, so the value of the question logit can also explain other things. For example, the N4 logit value is +0.07 and the N7 logit value is +0.21 , so it can be said that the difficulty level of the N7 question is three times the N4 question.

In Figure 1 there is also information about the logit mean value and the logit standard deviation values, 0 and 0.21 . Based on the average value and the standard deviation value, the level of difficulty of the instrument can be grouped [10]. The grouping of instrument difficulty levels is shown in Table 1 below.

Table 1. Instrument difficulty level grouping.

\begin{tabular}{ll}
\hline Range & Group \\
\hline Logit Value $>0,21$ & Difficult \\
$-0,21 \leq$ Logit Value $\leq 0,21$ & Medium \\
Logit Value $<-0,21$ & Easy \\
\hline
\end{tabular}

So based on Table 1, there is one difficult question, namely $\mathrm{N} 2$. There are six medium questions, namely N7, N4, N5, N3, and N6 questions. And there is one easy problem which is about N1.

\subsection{Student ability level (person measure)}

In addition to analyzing the difficulty level of the instrument, in the context of educational assessment also needs to be done an analysis of the level of student ability to work on the 
instrument. Analysis of student ability levels is useful for knowing students who have high, medium, and low ability levels in working on a given instrument. The analysis of the ability level of 29 students in working on the instruments on material number patterns can be seen in Figure 2 through detailed logit information (measure) of each student with the same scale. Data are displayed sequentially from the largest logit value to the smallest. A large logit score indicates a high level of student ability, while a small logit value indicates a low level of student ability. If the logit scores are the same, this shows the level of student ability is the same [6].

\begin{tabular}{|c|c|c|c|c|c|c|c|c|c|c|c|c|c|}
\hline Person: & $\begin{array}{r}\text { REAL SE } \\
\text { Person }\end{array}$ & $\begin{array}{l}\text { P. : } .41 \\
\text { STATIS }\end{array}$ & $\begin{array}{l}\text { REL. : } \\
\text { TICS: ME }\end{array}$ & $\begin{array}{l}14 \ldots \text { I } \\
\text { ASURE OF }\end{array}$ & $\begin{array}{l}\text { Item: } \\
\text { ORDER }\end{array}$ & REAL & SEP. & 1.32 & REL & $: .64$ & & & \\
\hline $\begin{array}{l}\text { | ENTRY } \\
\text { | NUMBER }\end{array}$ & $\begin{array}{l}\text { TOTAL } \\
\text { SCORE }\end{array}$ & $\begin{array}{l}\text { TOTAL } \\
\text { COUNT }\end{array}$ & MEASURE & $\begin{array}{l}\text { MODEL } \\
\text { S.E. }\end{array}$ & | IN IN & $\begin{array}{c}\text { IFIT } \\
\text { ZSTD }\end{array}$ & \begin{tabular}{|c|} 
OUT \\
|MNSQ
\end{tabular} & $\begin{array}{l}\text { FIT } \\
\text { ZSTD }\end{array}$ & $\begin{array}{l}\text { |PTMEAS } \\
\text { |CORR. }\end{array}$ & $\begin{array}{r}\text { SUR-AL | } \\
\text { EXP. | }\end{array}$ & \begin{tabular}{|l} 
EXACT \\
OBS\%
\end{tabular} & $\begin{array}{r}\text { MATCH } \\
\text { EXP\% }\end{array}$ & Person \\
\hline 23 & 40 & 8 & 1.85 & $1.39 \mid$ & MaXI & MUM ME, & EASURE & & .00 & $.00 \mid$ & 100.0 & $100.0 \mid$ & $23 \mathrm{~L}$ \\
\hline 3 & 38 & 8 & .96 & .44 & .45 & -.02 & .22 & $-.37 \mid$ & .68 & .17 & 87.5 & 79.8 & O3P \\
\hline 13 & 37 & 8 & .81 & $.35 \mid$ & .89 & $.27 \mid$ & .59 & $.10 \mid$ & .38 & .21 & 75.0 & $67.7 \mid$ & $13 \mathrm{~L}$ \\
\hline 16 & 37 & 8 & .81 & .35 & .64 & $-.01 \mid$ & .32 & $-.24 \mid$ & | .68 & .21 & 75.0 & 67.71 & $16 \mathrm{P}$ \\
\hline 26 & 37 & 8 & .81 & .35 & .48 & -.22 & .43 & -.09 & .31 & .21 & 75.0 & 67.7 & $26 \mathrm{~L}$ \\
\hline 5 & 36 & 8 & .71 & .301 & 1.18 & .49 & .77 & .18 & | .38 & .25 & 62.5 & 56.2 & $05 \mathrm{P}$ \\
\hline 6 & 34 & 8 & .56 & $.25[1$ & 1.57 & .94 & 2.02 & $1.17 \mid$ & -.08 & .30 & 50.0 & $44.6 \mid$ & $06 \mathrm{P}$ \\
\hline 4 & 33 & 8 & .50 & .24 & .58 & -.60 & .44 & -.59 & | .77 & .31 & 12.5 & 15.7 & $04 \mathrm{P}$ \\
\hline 9 & 33 & 8 & .50 & .24 & .71 & -.32 & .75 & -.06 & .14 & .31 & 12.5 & 15.7 & $09 \mathrm{~L}$ \\
\hline 20 & 33 & 8 & .50 & .24 & 1.60 & 1.03 & 1.90 & 1.14 & -.26 & $.31 \mid$ & .0 & 15.7 & $20 \mathrm{~L}$ \\
\hline 15 & 32 & 8 & .44 & $.22 i$ & .54 & $-.81 \mid$ & .40 & $-.82 \mid$ & .81 & $.33 \mid$ & 12.5 & $15.9 \mid$ & $15 \mathrm{P}$ \\
\hline 25 & 32 & 8 & .44 & .22 & 1.02 & .22 & .89 & .10 & .50 & .33 & 12.5 & 15.9 & $25 \mathrm{P}$ \\
\hline 29 & 32 & 8 & .44 & .22 & 1.24 & .59 & 1.60 & .93 & .19 & .33 & 12.5 & 15.9 & $29 \mathrm{P}$ \\
\hline 7 & 31 & 8 & .40 & .211 & 1.39 & .85 & 1.75 & 1.13 & -.16 & .34 & .0 & 15.8 & $07 \mathrm{~L}$ \\
\hline 21 & 30 & 8 & .35 & $.21 \mid$ & .72 & -.51 & .65 & -.43 & .65 & .36 & 37.5 & 16.6 & $21 \mathrm{~L}$ \\
\hline 14 & 29 & 8 & .31 & .20 & .87 & -.15 & .98 & .16 & $\begin{array}{l}\text { I } \\
\end{array}$ & .37 & 25.0 & 16.8 & $14 \mathrm{P}$ \\
\hline 11 & 28 & 8 & .27 & .19 & 1.34 & .87 & 1.67 & 1.25 & --.37 & $.37 \mid$ & 25.0 & 7.21 & $11 \mathrm{~L}$ \\
\hline 19 & 28 & 8 & .27 & .19 & .78 & -.42 & .72 & -.43 & .40 & .37 & 12.5 & 7.2 & $19 \mathrm{P}$ \\
\hline 10 & 27 & 8 & .24 & .19 & .89 & $-.17 \mid$ & .91 & -.04 & .42 & .38 & 25.0 & 7.21 & $10 \mathrm{P}$ \\
\hline 18 & 27 & 8 & .24 & .19 & 1.19 & .58 & 1.96 & $1.73 \mid$ & .20 & .38 & .0 & 7.21 & $18 \mathrm{P}$ \\
\hline 17 & 26 & 8 & .20 & .19 & .84 & -.32 & .85 & -.21 & .44 & .38 & 12.5 & 8.5 & $17 \mathrm{P}$ \\
\hline 1 & 25 & 8 & .17 & .18 & 1.32 & .94 & 1.31 & .81 & .08 & .39 & 25.0 & 8.5 & $01 \mathrm{~L}$ \\
\hline 24 & 25 & 8 & .17 & .18 & .69 & $-.86 \mid$ & .65 & $-.83 \mid$ & .45 & .39 & 37.5 & 8.5 & $24 \mathrm{P}$ \\
\hline 27 & 25 & 8 & .17 & .18 & 1.18 & .61 & 1.07 & $.31 \mid$ & .55 & .39 & .0 & $8.5 \mid$ & $27 \mathrm{~L}$ \\
\hline 8 & 24 & 8 & .13 & .18 & .94 & -.08 & .90 & $-.14 \mid$ & .47 & .39 & .0 & 7.71 & $08 \mathrm{~L}$ \\
\hline 28 & 20 & 8 & .00 & .18| & .93 & $-.13 \mid$ & .88 & -.25 & .47 & .39 & .0 & 9.1 & $28 \mathrm{P}$ \\
\hline 2 & 19 & 8 & -.03 & .18 & 1.27 & .91 & 1.20 & .64 & .37 & .39 & .0 & $8.7 \mid$ & $02 \mathrm{~L}$ \\
\hline 12 & 17 & 8 & -.09 & .18 & .80 & -.56 & . .75 & $-.59 \mid$ & .66 & .38 & .0 & 9.6 & $12 \mathrm{~L}$ \\
\hline 22 & 15 & 8 & -.16 & .18 & 1.24 & $.76 \mid$ & 1.16 & $.49 \mid$ & | .49 & $.37 \mid$ & .0 & $10.6 \mid$ & $22 \mathrm{~L}$ \\
\hline & 29.3 & 8. & .4 & .2 & .97 & .11 & .9 & $.2 \mid$ & & & 6 & $22.7 \mid$ & \\
\hline 8.5 & 6.3 & .0 & .39 & .22 & .32 & $\begin{array}{l}.1 \\
.61\end{array}$ & .50 & .71 & & & 27.0 & 22.41 & \\
\hline
\end{tabular}

Fig. 2. Student Ability Level.

Based on Figure 2, students who have the highest level of ability are 23L students with a logit score of +1.85 . While students who have the lowest level of ability are $22 \mathrm{~L}$ students with a logit value of -0.16 . Students $11 \mathrm{~L}$ and $19 \mathrm{P}$ have the same logit value of +0.27 , this shows the level of ability of the two students are the same.

The resulting scale has the same distance, so the student logit value can also explain other things. For example, student $17 \mathrm{P}$ 's logit score is +0.20 and student $07 \mathrm{~L}$ 's logit value is +0.40 , so the student's $07 \mathrm{~L}$ ability level is twice that of 17P's student, in the context of being able to work on the question.

In Figure 2 there is also information about the logit mean value and the standard deviation value of logit, 0.41 and 0.39 . Based on the average value and the standard deviation value, the ability level of students can be grouped [10]. The grouping of student ability levels is shown in the following Table 2. 
Table 2. Grouping Student Ability Levels.

\begin{tabular}{ll}
\hline Range & Group \\
\hline Logit value $>0,80$ & High \\
$0,02 \leq$ Logit value $\leq 0,80$ & Medium \\
Logit value $<0,02$ & Low \\
\hline
\end{tabular}

So based on Table 2, there are five students who belong to the group of high-ability students, namely students 23L, 03P, 13L, 16P, and 26L. There are sixteen students included in the group of moderately capable students, namely students $05 \mathrm{P}, 06 \mathrm{P}, 04 \mathrm{P}, 09 \mathrm{~L}, 20 \mathrm{~L}, 15 \mathrm{P}, 25 \mathrm{P}$, 29P, 07L, 21L, 14P, 11L, 19P, 10P, 18P, and 17P. And there are eight students who belong to the group of low-ability students, namely students 01L, 24P, 27L, 08L, 28P, 02L, 12L, and 22L.

\subsection{Wright map analysis (Person-Item Map)}

One of the strengths of the Rasch model is that it produces a Wright map that illustrates the distribution of student ability levels and instrument difficulty levels on the same scale [6], [11]. The following is a Wright map that illustrates the distribution of the ability levels of 29 students and the distribution of the difficulty level of the material instrument of number patterns.

Map of Wright on the left in Figure 3 shows the ability level of 29 students from the highest to the lowest. 23L students when compared with other students the level of ability is the highest with a logit value above 1 . In addition, $23 \mathrm{~L}$ students also have a high level of ability that is different (outlier) because the logit value is more than the limit of two standard deviations (T). Whereas 22L students when compared with other students the level of ability is the lowest with a logit value below 0 . In addition, there are also four students who have the same level of ability namely $10 \mathrm{P}, 11 \mathrm{~L}, 18 \mathrm{P}$, and $19 \mathrm{P}$ with a logit value above 0 .

The Wright map on the right shows the difficulty level of the questions in the material pattern of numbers from the highest (difficult) to the lowest (easy). Problem N2 has the highest difficulty level (difficult) with a logit value above 0 , meaning that the probability of all students to work on this problem correctly is small. While N1 questions have the lowest difficulty level (easy) with a logit value below 0 , meaning that almost all students can work on this problem correctly. In addition, there are two questions with the same difficulty level, namely N3 and N6 questions.

There are thirteen students $(23 \mathrm{~L}, 03 \mathrm{P}, 13 \mathrm{~L}, 16 \mathrm{P}, 26 \mathrm{~L}, 05 \mathrm{P}, 06 \mathrm{P}, 04 \mathrm{P}, 09 \mathrm{~L}, 15 \mathrm{P}, 20 \mathrm{~L}, 25 \mathrm{P}$, and 29P) who can work on all N2 questions. This is because the ability level of the thirteen students is above the difficulty level of $\mathrm{N} 2$ questions. This can be seen from the 13th-grade logit of the student is more than the N2 logit value. The student's logit value is greater than the value of the item's logit meaning the probability to work on the problem correctly is more than $50 \%$. Thirteen students will get the maximum value that can be obtained

The level of ability of 17P students is the same as the level of difficulty of N7 questions. This can be seen from the 17P student's logit value is the same as the N7's question logit value. The student logit score is the same as the value of the logit about the meaning of the probability to do the problem correctly is $50 \%$.

There are four students $(01 \mathrm{~L}, 08 \mathrm{~L}, 24 \mathrm{P}, 27 \mathrm{~L})$ who cannot work on N7 problems because the level of ability of the four students is below the difficulty level of N7 questions. This can be seen from the value of the fourth student logit below the logit value of question N7. The student's 
logit score is less than the value of the item's logit meaning the probability to work on the problem correctly is less than $50 \%$.

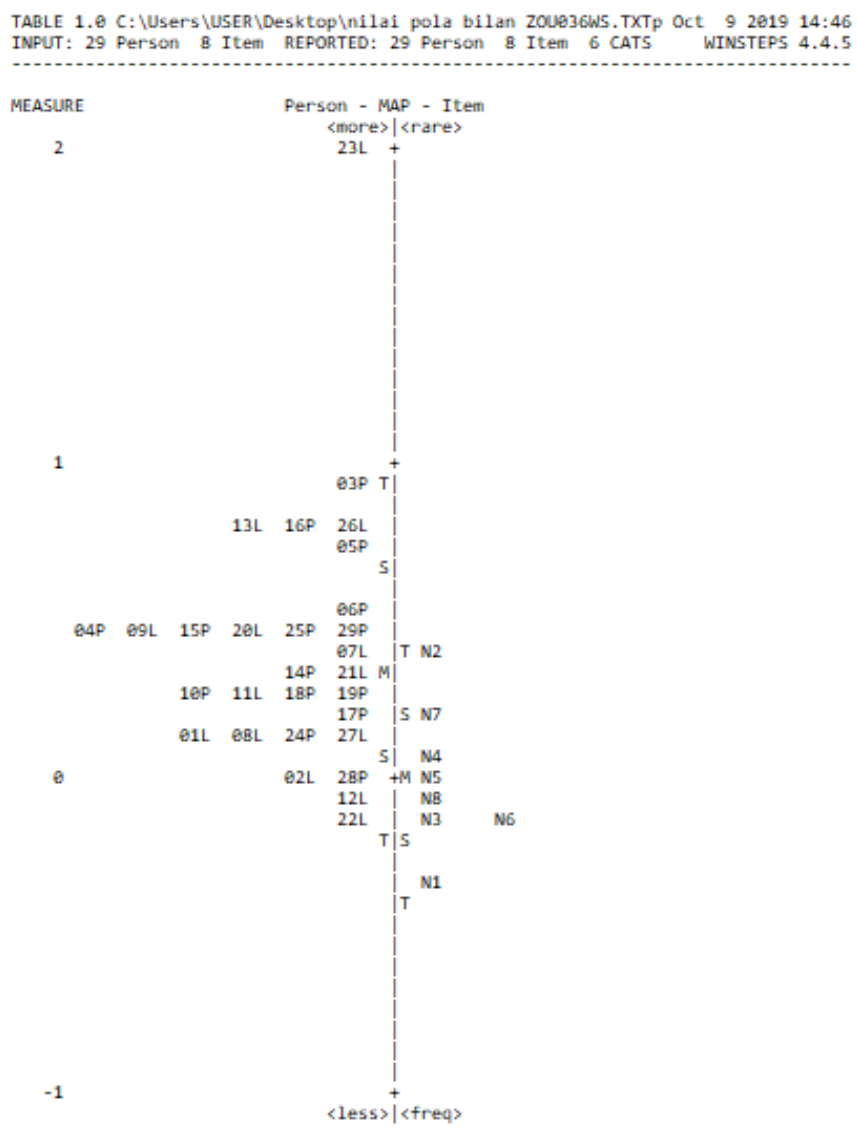

Fig. 3. Wright Map.

When compared to the distance between the M-S-T (mean, 1 SD, and 2 SD) on the Wright map, it can be seen that the distribution for students' level of ability (left) is wider than the distribution for instrument difficulty level (right). This shows that the level of difficulty of the diversity instruments is not far adrift, but the level of student ability varies.

The logit value of the average level of difficulty of the instrument is 0 , while the logit value of the average level of student ability is above 0 . So the average value of the level of student ability is greater than the average value of the level of difficulty of the instrument. This shows that the average level of student ability is above the average level of difficulty of the instrument

The Wright map analysis can provide information about the quality of the instruments being tested. So that information can be obtained about the questions which many students successfully work on and the questions which many students fail to work on. So that we can provide assistance to students who need help.

\subsection{Instrument analysis}


In the Rasch model, instrument analysis can be carried out in more detail in the form of statistical summaries. The statistical summary provides information about the overall quality of student response patterns, the quality of the instruments used, and the interaction between students and instruments [6]. The following is a statistical summary of 29 students who answered 8 material number material instruments presented in Figure 4.

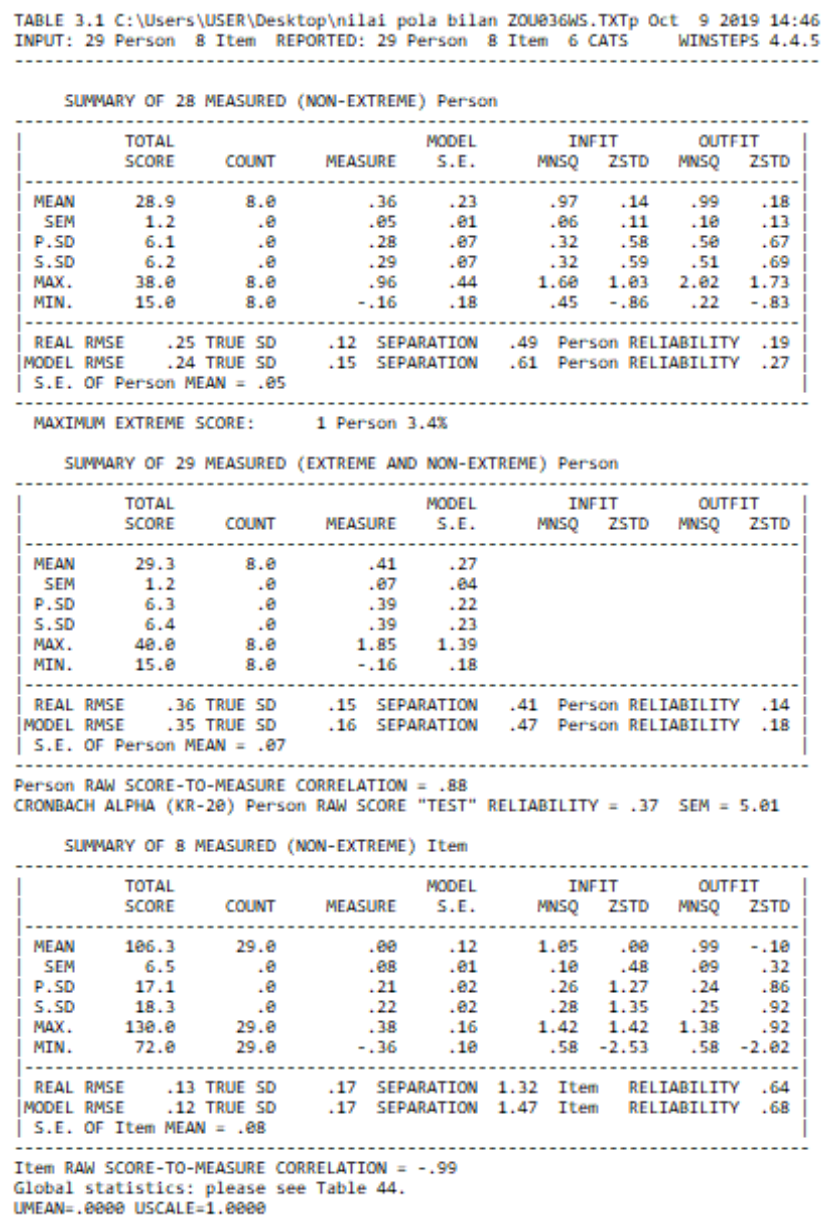

Fig. 4. The statistical summary.

Based on Figure 4 person measure value is +0.41 which shows the average value of all students working on the given instrument. The average value that is greater than the logit value of 0 indicates a tendency for students to have a greater ability than the instrument difficulty level. INFIT MNSQ and OUTFIT MNSQ, for the person table the average values are 0.97 and 0.99 , the ideal value is 1 (the closer to 1 the better). In addition, for INFIT ZSTD and OUTFIT ZSTD the average values are respectively 0.14 and 0.18 , the ideal value is 0 (the closer to 0 the better). Likewise the MNSQ INFIT and MNSQ OUTFIT for the item table, the average values are 1.05 and 0.99 , the ideal value is 1 (the closer to 1 the better). In addition, for INFIT ZSTD and OUTFIT ZSTD the average values are respectively 0 and -0.10 , the ideal value is 0 (the 
closer to 0 the better). Cronbach's alpha value of 0.37 shows the interaction between students and the instrument as a whole is bad. In addition, the value of person reliability is +0.14 and item reliability is +0.64 . So it was concluded that the consistency of students' answers was weak, but the quality of the instruments was sufficient.

\section{Conclusion}

Based on the results of the analysis using the Rasch model, it was found that there was one question that was included in the difficult questions group, six questions that were included in the medium questions group, and one question that was included in the easy questions group. In addition, there are five students who belong to the high student group, sixteen students who belong to the medium student group, and eight students who belong to the low student group. The level of difficulty about diversity is not far adrift, but the level of student ability varies. The average level of student ability is above the average level of difficulty of the questions. The consistency of answers from students is weak, but the quality of the questions is sufficient.

\section{References}

[1] NCTM.: Principles and Standards for SchoolnMathematics. Reston (2000).

[2] Fey, J. T. And Smith D. A.: Algebra as Part of an Integrated High School Curriculum. And the Rest is Just Algebra. Springer (2017).

[3] Kieran, C.: Seeking, Using, and Expressing Structure in Numbers and Numerical Operations: A Fundamental Path to Developing Early Algebraic Thinking. Teaching and Learning Algebraic Thinking with 5 - to 12 - Year - Olds. Springer (2018).

[4] Wilkie, K. J. and Clarke, D. M.: Developing Students' Functional Thinking in Algebra through Different Visualisations of a Growing Pattern's Structure. Vol. 28., pp. 223-243. Mathematics Education Research Journal (2016).

[5] Curtin, M., et al.: The Early Development Instrument: an evaluation of its five domains using Rasch analysis. Vol. 16., pp. 1-14. BMC Pediatrics (2016).

[6] Sumintono, B. and Widhiarso, W.: Aplikasi Pemodelan Rasch pada Assessment Pendidikan. Trim Komunikata (2015).

[7] Andrich, D.: Georg Rasch and Benjamin Wright's Struggle With the Unidimensional Polytomous Model With Sufficient Statistics. Vol. 76., pp. 713-723. Educational and Psychological Measurement (2016).

[8] Sumintono, B. and Widhiarso, W.: Aplikasi Model Rasch untuk Penelitian Ilmu-Ilmu sosial. Trim Komunikata (2014).

[9] Pang, J. S. And Kim, J. W.: Characteristics of Korean Students' Early Algebraic Thinking: A Generalized Arithmetic Perspective. Teaching and Learning Algebraic Thinking (2018).

[10] Arikunto, S.: Dasar-dasar Evaluasi Pendidikan. Bumi Aksara (2009).

[11] Van Zile-Tamsen, C.: Using Rasch Analysis to Inform Rating Scale Development. Vol. 58., pp. 922-933. Research in Higher Education (2017). 